\title{
A WORKFLOW FOR GEOMETRIC COLOUR PHOTOGRAPHY OF PAINTED SURFACES
}

\author{
A. Dhanda ${ }^{1, *}$, G. Scarpa ${ }^{2}$, S. Fai ${ }^{1}$, M. Santana Quintero ${ }^{1}$ \\ ${ }^{1}$ Carleton Immersive Media Studio (CIMS), Carleton University, Ottawa, Canada - (adhanda, sfai)@ cims.carleton.ca, \\ (mario.santana)@ carleton.ca \\ ${ }^{2}$ The Factum Foundation, Madrid, Spain - gabriel@factum-arte.com
}

KEY WORDS: Painting, Photography, Photogrammetry, Orthophoto, 16-bit, Colour, Archiving

\begin{abstract}
:
Colour fidelity is vital when documenting painted surfaces. The 2.5D nature of many painted surfaces makes orthophotos and digital surface models (DSMs) common products of the documentation process. This paper presents a workflow to combine photographic and photogrammetric methods to produce aligned colour and depth (orthophotos and DSMs). First, two photogrammetric software (Agisoft Photoscan and Capturing Reality Reality Capture) were tested to determine if they adjusted the colour data during the processing stages. It was found that Photoscan can produce 16-bit orthophotos without manipulating the data; however, Reality Capture is currently limited to 8-bit results. When capturing a surface using photogrammetry, it is common to use the same data for colour and depth. The presented workflow, however, argues that better colour accuracy can be achieved by capturing the two datasets separately and combining them in photogrammetric software. The workflow is demonstrated through the documentation of an unnamed religious painting from the $17^{\text {th }}$ century.
\end{abstract}

\section{INTRODUCTION}

The digitization of paintings for archiving often involves photography - ranging from the infrared (IR) to the visible (VIS), and finally to the ultraviolet (UV) spectrum - and $2.5 \mathrm{D}$ capture of the painting surface. This digital documentation can be used for investigations, as a record, for monitoring the condition of the surface, etc. Photogrammetry is a popular choice for geometric surface capture because it is cheaper and more widely accessible than other methods. When dealing with painted surfaces, it is critical that the colour information accurately represents the subject. Photography not only tells conservators about the visual condition of a wall painting but can also give insights as to the physical and chemical compositions of the materials (Verri, 2008; Verri, 2009). This makes it essential for practitioners to understand how colour information is processed and how best to integrate it in the photogrammetric pipeline - when combining photographic and photogrammetric techniques to produce colour orthophotos or 3D models. However, there is a gap of knowledge as to how many commercial ('black box') photogrammetric software manipulate colour data. In this paper, we compare the colour processing accuracy of two photogrammetric programs (Agisoft Photoscan (2018) and Capturing Reality Reality Capture (2018)). Also, it proposes a workflow for combining photographic and photogrammetric techniques to produce a digital surface model (DSM) and orthophoto of a painted surface for digital archiving and monitoring.

\subsection{Bit Depth in Digital Images}

When one takes a photograph, a digital camera sensor receives electromagnetic radiation from its environment. The analogueto-digital converter (ADC) of the camera changes the signals received by the camera into discrete values through a process called quantization. A pixel value is rounded to the closest matching discrete value available in the ADC's tonal range. This tonal range represents all the possible values of a pixel and can be calculated by $2^{\mathrm{N}}$, where $\mathrm{N}$ is the bit-depth/colour channel of the ADC. For example, an 8-bit pixel can have one of 256 tones, while a 16-bit pixel can have one of 65536 tones. When looking at a whole image with a depth of 8-bits, it is said that it has an overall image depth of 24-bits - one per colour channel (Verhoeven, 2016). However, this image is usually referred to as an 8-bit image, not a 24-bit image; the 'per channel' part is assumed. Most DSLR cameras capture RAW information at 12bits or 14-bits.

\section{RELATED WORKS}

Warda et al. (2011) introduced a guide for conservation photography. Dyer et al. (2013) introduced a standardized workflow for capturing and processing multi-spectral imaging to ensure consistent results during repeated photography of paintings. On the subject of combining multiple photographic documentation methods, Remondino et al. (2011) reviewed how different technologies - terrestrial laser scanning, triangulation scanning, and photogrammetry - had been used to document the surfaces of various paintings. Abate et al. (2014) used structured light scanning and photogrammetry to document the surfaces of several paintings. The photogrammetry was used to produce point cloud data, DSMs, and colour orthophotos of the paintings. Nocerino et al. (2018) combined VIS and UV photogrammetry to capture the details of a Greek vase. In these related works, the research focused on the capture and rendering of objects - colour fidelity was not addressed in the digital workflows. Aure et al. (2017) combined high fidelity colour with painting geometry but used separate capture methods - a triangulation scanner and albedo capture from reflectance transformation imaging (RTI) which were aligned using manually selected control points. This paper aims to address the gap between photographic and photogrammetric approaches by suggesting a workflow that will produce geometric information aligned with precise colour at a sufficient bit depth for archiving.

\section{VIS COLOUR IN PHOTOGRAPHIC IMAGES}

Visible colour - ranging from 360 to $780 \mathrm{~nm}$ - is separated into models, which are different ways that we represent colour - ex)

\footnotetext{
* Corresponding author
} 
RGB, XYZ, and L*a*b*. These models are all trichromatic systems, which means that any colour in the system is made by additively mixing the three set primaries for that colour model. In 1931, the Commission Internationale de l'Éclairage (CIE) introduced the XYZ colour model. The model set a new standard for scientific colour representation by creating three imaginary primaries $(\mathrm{X}, \mathrm{Y}$, and $\mathrm{Z})$ that were set so that no colour values would be negative (Wyszecki and Stiles, 1982). In 1976 the CIE introduced the $\mathrm{L} * \mathrm{a} * \mathrm{~b} *$ colour model, which has since become the standard for colour representation. These colour models are further separated into colour spaces, which are profiles within the models that can represent a certain range of colour or gamut - ex) the RGB colour model has colour spaces like sRGB, Adobe RGB (1998), and ProPhoto (Westland et al., 2012; Verhoeven, 2016). Figure 1 shows the sRGB, Colormatch RGB, Adobe RGB (1998), ProPhoto RGB, and SWOP CMYK colour spaces projected onto the 1931 CIE chromacity diagram - a 2D projection of the XYZ colour model. See Wyszecki and Stiles (1982), and Verhoeven (2016) for more information on the CIE colour models.

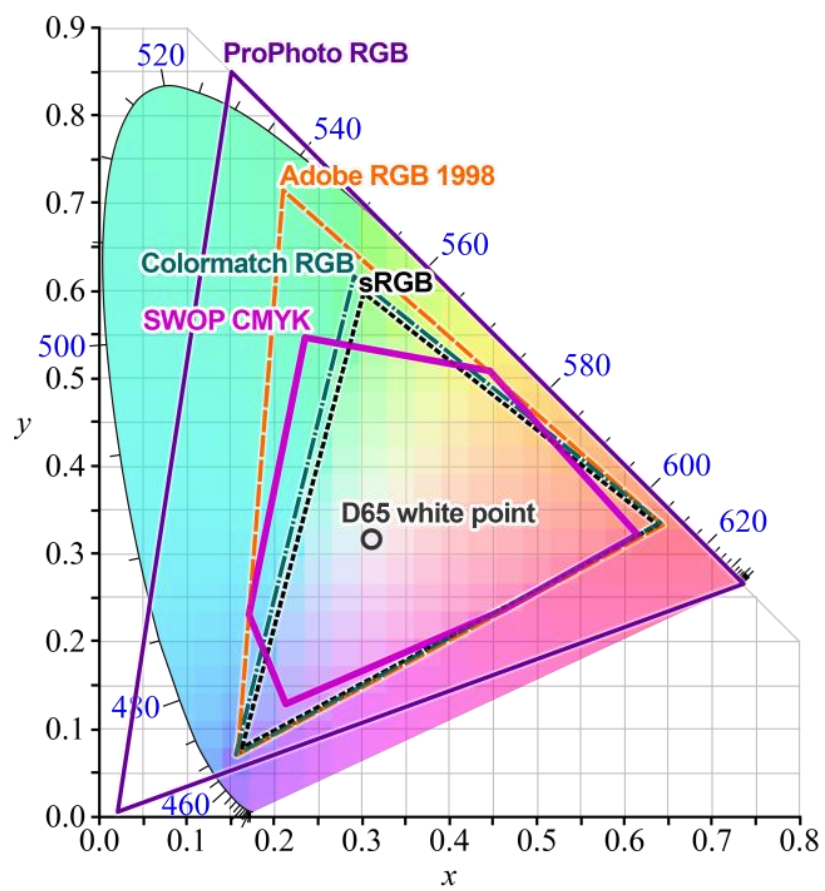

Figure 1. Colour spaces projected onto the $1931 \mathrm{CIE}$ chromacity diagram (Wikimedia Commons contributors, 2014)

Dyer et al. (2013) introduced a workflow that uses a Macbeth colour checker chart, and a uniformly reflective board to correct images for exposure, colour representation, and inhomogeneous lighting in VIS photography of painted surfaces They also present workflows for UV and IR images, but they involve additional equipment. The proposed workflow uses nip2, which is a graphical user interface (GUI) for the free image processing system known as VIPS. The workflow involves five main steps:

1) The RAW images are converted to linear RGB TIFF images with a depth of 16-bits

2) The 16-bit images are imported into the workspace made for nip 2 - the colour checker image, the uniform reflective board image, and the image to be corrected

3) The user identifies the 24-colour grid of the colour checker, and selects the type of correction - ex) colour and exposure
4) nip2 converts the linear RGB image to the $X Y Z$ colour model, corrects the illumination inhomogeneities, colour response and exposure and calculates the average colour error from known values $(\Delta \mathrm{E})$

5) The 8 or 16-bit corrected image is exported in the sRGB colour space

Dyer et al. (2013) recommend using a uniformly reflective board to correct for inhomogeneous lighting distribution - a technique known as 'flat fielding'. However, it is not practical to have a uniformly reflective board that matches every size of painting that could be photographed. We use a more widely applicable, but simplified, flat fielding method that is demonstrated in the example shown in this paper. Instead of using the standard reflective board, a colour checker is photographed in the middle and corners of the piece. The colour correction and initial illumination correction in nip2 are done based on the colour checker chart in the middle of the piece. At the end of the orthophoto process (described in Section 5), the difference of RGB values is calculated between the greyscale patches of central colour checker chart and edge greyscale patches. The differences in values are used to create a gradient to correct for the changes in exposure (Figure 2). This does not provide an absolute correction, as a uniformly reflecting board would, but rather assumes that the exposure in the centre of the painting is correct and makes corrections at the edges - where most falloff occurs - to achieve an approximate flat fielding result.


Figure 2. The colour checkers placed on the painting (top), and the gradient mask of the top-right colour checker for the simplified flat field correction (bottom) 


\subsection{Bit Depth and Archiving}

16-bit images are ideal for the archiving of digital images. 16-bit images can hold more tonal information which is essential when managing colour in colour spaces with a larger gamut - ex) ProPhoto (Berns, 2014; Verhoeven, 2016). In addition, 8-bit images cannot withstand edits the way 16-bit images can; digital edits can degrade the limited information and cause banding effects to be visible in the image (Figure 3).

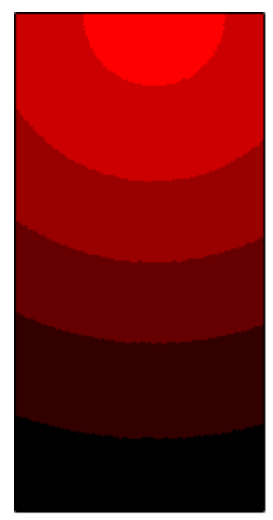

8-bit gradient

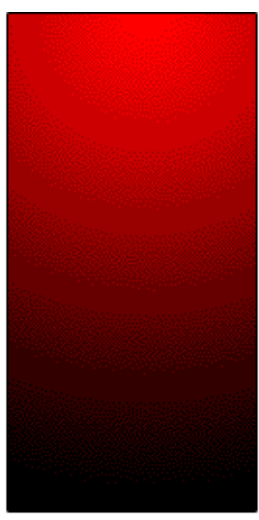

8-bit gradient, dithered

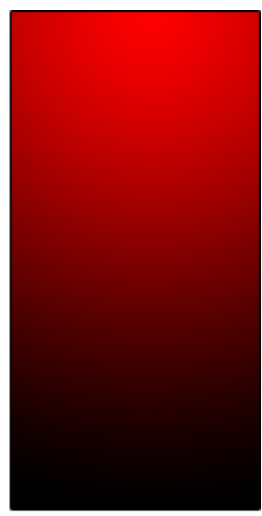

24-bit gradient
Figure 3. Colour banding in an 8-bit gradient compared to a 24bit gradient (Wikimedia Commons contributors, 2004)

\section{COLOUR IN PHOTOGRAMMETRIC SOFTWARE}

When working with painted surfaces-and in other situations where colour consistency is desired-it is important to understand how the calibrated colour images are affected by the photogrammetric software. Does the colour remain consistent between input and output? To test this, three images of a colour checker chart were colour corrected using the method described in Dyer et al. (2013) - one at 8-bits, one at 16-bits, and one at 32bits. The 8-bit and 16-bit images were created from applying the workflow described in Section 3 to the RAW images from the camera. The 32-bit image was made from converting the corrected 16-bit image to a linear 32-bit image in Photoshop. The images were then aligned in a bundle adjustment with a set of photogrammetric images in two different photogrammetric software (Agisoft Photoscan 1.4.3 (2018), Reality Capture 1.0.3 (2018)). The colour checker images were projected onto the surface producing the orthophotos used to compare the values before and after photogrammetric processing.

The orthophotos and original colour corrected image were manually aligned in Photoshop. A colour picker, with an area of 51 pixels $^{2}$ was used to check the RGB values in each square of the colour checker chart. The average of the larger area helps to account for alignment/point picking errors between the images. The errors were then normalized to a $0-1$ range to allow comparison between the results from different bit depths - the tonal errors can be seen in the Appendix. Table 1 shows the results of the 8-bit comparison. It is evident from Table 1 that the output data from both Photoscan and Reality Capture is very similar to the original input data. The errors from reality capture are minimal-less than one-tenth of a percent, or one tone value out of the possible 256 - that they are probably due to alignment/point picking errors.

\begin{tabular}{|c|c|c|c|c|c|c|}
\hline & \multicolumn{3}{|c|}{ Photoscan (8-bit) } & \multicolumn{3}{c|}{ Reality Capture (8-bit) } \\
\hline & $\mathrm{R}$ & $\mathrm{G}$ & $\mathrm{B}$ & $\mathrm{R}$ & $\mathrm{G}$ & $\mathrm{B}$ \\
\hline$\mu_{\text {Error }}$ & 0.000 & 0.000 & 0.000 & -0.001 & 0.000 & 0.000 \\
\hline$\sigma_{\text {Error }}$ & 0.000 & 0.000 & 0.000 & 0.001 & 0.001 & 0.001 \\
\hline
\end{tabular}

Table 1. 8-bit colour errors (measured in tones) of a colour chart measured before and after processing the images in Agisoft

Photoscan (2018) and Capturing Reality Capture (2018)

Table 2 shows the results of the 16-bit test. The output from Reality Capture was an 8-bit image, despite the input of the 16bit data. As a result, the comparison was made between the Reality Capture 8-bit data and the corrected 8-bit data that was used for the comparison shown in Table 1. Though the tonal colour errors are higher in the Photoscan 16-bit test (see the Appendix), they are the same as the 8-bit results when normalized. This supports the conclusion that the errors were likely alignment/point picking errors. The Reality Capture output, however, has substantial errors. It is clear from visual inspection of the image that the internal conversion from 16-bits to 8-bits in Reality Capture adds heavy saturation to the image.

\begin{tabular}{|c|c|c|c|c|c|c|}
\hline & \multicolumn{3}{|c|}{ Photoscan (16-bit) } & \multicolumn{3}{c|}{ Reality Capture (8-bit) } \\
\hline & $\mathrm{R}$ & $\mathrm{G}$ & $\mathrm{B}$ & $\mathrm{R}$ & $\mathrm{G}$ & $\mathrm{B}$ \\
\hline$\mu$ Error & 0.000 & 0.000 & 0.000 & 0.105 & 0.085 & 0.107 \\
\hline бError & 0.000 & 0.000 & 0.000 & 0.107 & 0.047 & 0.142 \\
\hline
\end{tabular}

Table 2. 16-bit colour errors (measured in tones) of a colour

chart measured before and after processing the images in Agisoft Photoscan (2018) and Capturing Reality Capture (2018)

For the 32-bit test, the images were linearly converted down to 16-bits before the RGB values of the patches were sampled; the results can be seen in Table 3. The output from Reality Capture was an 8-bit image, despite the input of the 32-bit data. As a result, the comparison was made between the Reality Capture 8bit data and the corrected 8-bit data that was used for the comparison shown in Table 1. The errors from both Photoscan and Reality Capture are low, and consistent with expected alignment/point picking errors.

\begin{tabular}{|c|c|c|c|c|c|c|}
\hline & \multicolumn{3}{|c|}{ Photoscan (16-bit) } & \multicolumn{3}{c|}{ Reality Capture (8-bit) } \\
\hline & $\mathrm{R}$ & $\mathrm{G}$ & $\mathrm{B}$ & $\mathrm{R}$ & $\mathrm{G}$ & $\mathrm{B}$ \\
\hline$\mu_{\text {Error }}$ & 0.000 & 0.000 & 0.000 & -0.001 & 0.000 & 0.000 \\
\hline$\sigma_{\text {Error }}$ & 0.000 & 0.000 & 0.000 & 0.001 & 0.001 & 0.001 \\
\hline
\end{tabular}

Table 3. 32-bit colour errors (measured in tones) of a colour chart measured as 16-bit images before and after processing the images in Agisoft Photoscan (2018) and Capturing Reality Capture (2018) as 32-bit images

From our analysis, it is evident that Photoscan 1.4.3 can produce an orthophoto at a bit depth $(8,16$, or 32$)$ that remains faithful to the original input images. Reality Capture 1.0.3, however, can only produce high fidelity images at a depth of 8-bits.

\section{WORKFLOW}

As shown in Section 4, it is possible to produce 16-bit orthophotos aligned with depth in Photoscan. The workflow to achieve this (outlined in Figure 4) is described in the following sections. An unnamed religious painting from the $17^{\text {th }}$ century is used to demonstrate the workflow through visible-reflected (VIS) photography - though it can be applied to photography of other spectrums of light as well. 


\subsection{Data Capture}

The data capture of the photogrammetry and colour photography is done separately to ensure ideal results for each. At the painting scale, photogrammetry is typically captured from a close distance (i.e. less than two metres), though this will change depending on the required ground sample distance (GSD). The nature of structure from motion (SfM) photogrammetry necessitates that the capture comes from varying camera positions. This leads to better geometric measurement, but not better colour reproduction. Taking images with varying positions (with the same lighting) can lead to issues such as glaring artifacts. Varying the light positions along with the camera, to avoid glaring artifacts, runs the risk of inhomogeneous illumination.

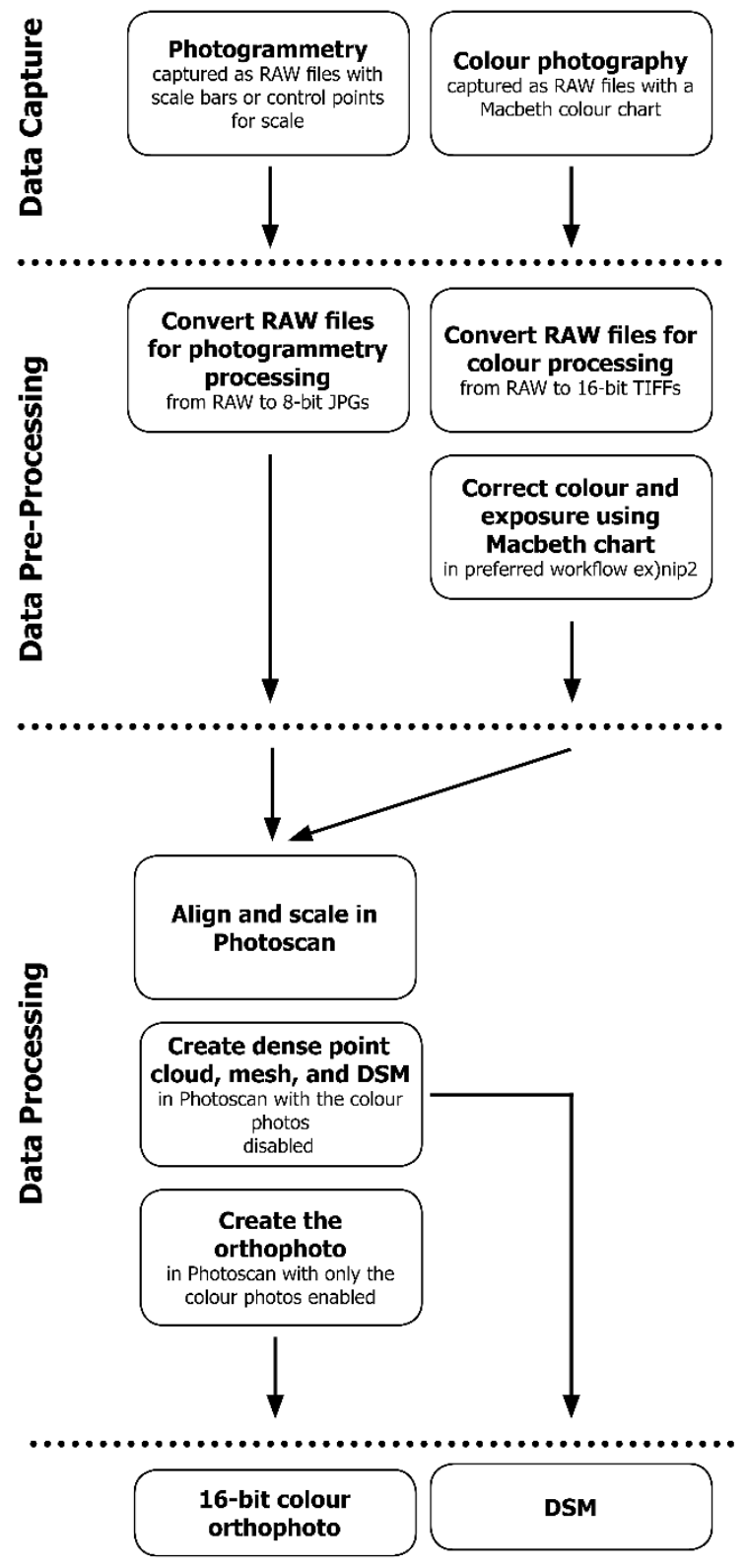

Figure 4. The workflow to produce a DSM and 16-bit colour orthophotos

Painting photography is typically captured from a single vantage point, and a far distance; this is for two reasons: to prevent perspective effects and to avoid glare. These artifacts occur when the illumination is scattered off the painting surface and - on highly specular surfaces - returns to the camera without losing too much intensity. For example, colour photography - which is captured from a single point in a single image, or a mosaic of images (Figure 5) - is not captured in a proper network for surface reconstruction. Compared to colour photography, photogrammetry is captured closer to the object and in varying positions, which can result in some veiling glare in the colour capture (Figure 6).

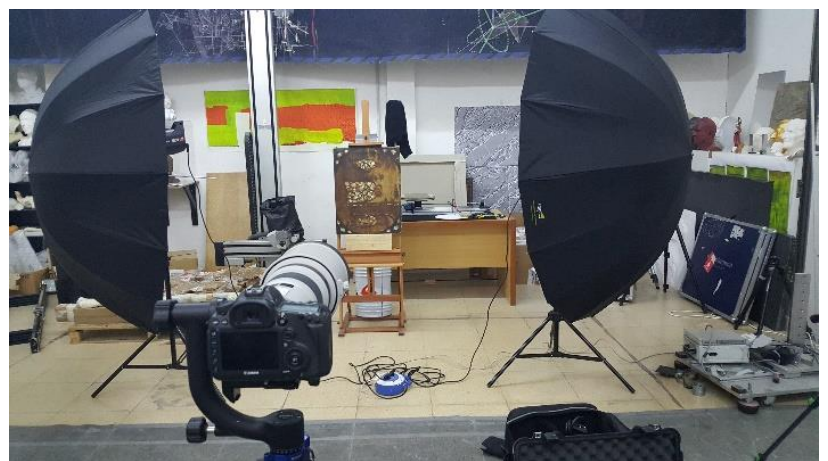

Figure 5. Colour photography capture setup for the unnamed $17^{\text {th }}$-century painting

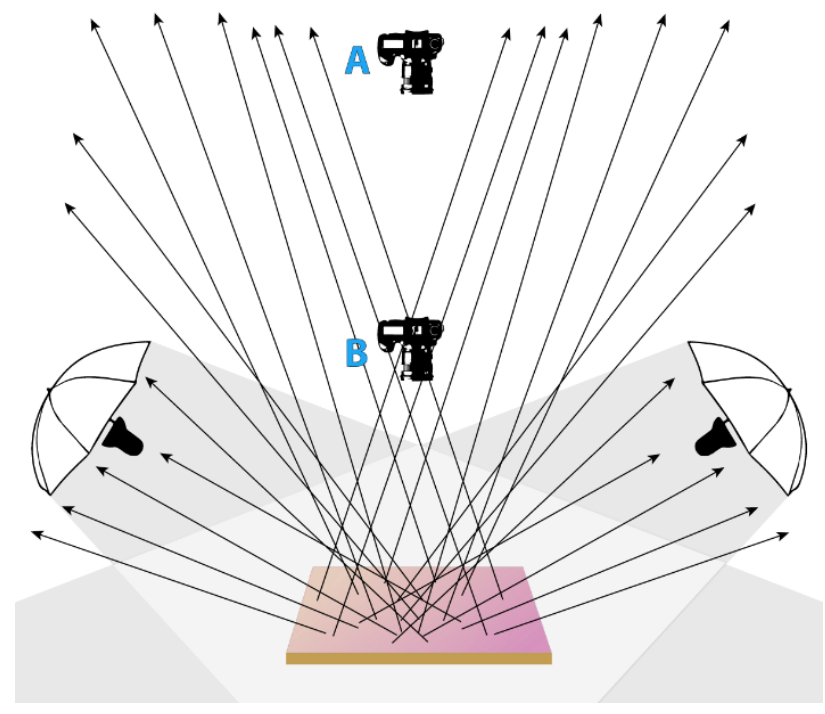

Figure 6. Positions of photographic (A) vs. photogrammetric (B) setups in the capture of painted surfaces

The unnamed $17^{\text {th }}$-century painting was photographed using a Canon 5DS R and a Canon $300 \mathrm{~mm} \mathrm{f} / 2.8 \mathrm{~L}$ lens. The camera was placed $3.5 \mathrm{~m}$ away from the painting resulting in a photographic ground sample distance (GSD) of just under $50 \mu \mathrm{m}$. The photogrammetry of the painting was done with the 5DS R and a Sigma $50 \mathrm{~mm}$ f/1.4 DG lens. The photogrammetry was captured from $0.75 \mathrm{~m}$ away from the painting at a nominal GSD of just over $60 \mu \mathrm{m}$.

\subsection{Data Pre-Processing}

The RAW images from the camera are pre-processed separately according to their capture method. 
5.2.1 Photogrammetric Images: The photogrammetric images are converted to high-quality 8-bit JPEG images; the accuracy of the image colour in these photos is not important since they are only needed to calculate the geometry of the subject.

5.2.2 Colour Photography Images: The RAW colour images are converted to 16-bit TIFF images that are corrected for exposure, white balance, and colour representation. The colour photos of the painting were corrected using the method described in Section 3.

\subsection{Data Processing}

The photogrammetric and colour images are aligned together in Agisoft Photoscan 1.4.3 using a self-calibrating bundle adjustment. The colour photography images are then disabled during the dense image matching (DIM) and digital surface model (DSM) creation. Then, all images except for the colour photography images are disabled, and an orthophoto is created. The orthophoto is exported as an uncompressed TIFF file at 16bits. The result is an orthophoto and matching DSM that can be used for monitoring, visualization, or archiving. The final orthophoto resulting from the workflow is presented in Figure 7, along with renders of the colour and depth data.

\section{CONCLUSION}

Our research has developed a workflow for creating high-quality orthophotos for archiving by capturing photographic and photogrammetric data separately. This separation allows for optimal photogrammetric positioning, without sacrificing colour fidelity. In the workflow, Agisoft Photoscan is recommended because it does not alter colour data or bit depth during processing.

Future work involves testing the colour fidelity of Photoscan with colour spaces that have a larger gamut, such as ProPhoto.

\section{ACKNOWLEDGEMENTS}

The authors would like to thank the Factum Foundation for Digital Conservation for their support and guidance during this project. This work was supported by the NSERC Create Heritage Engineering grant at Carleton University.

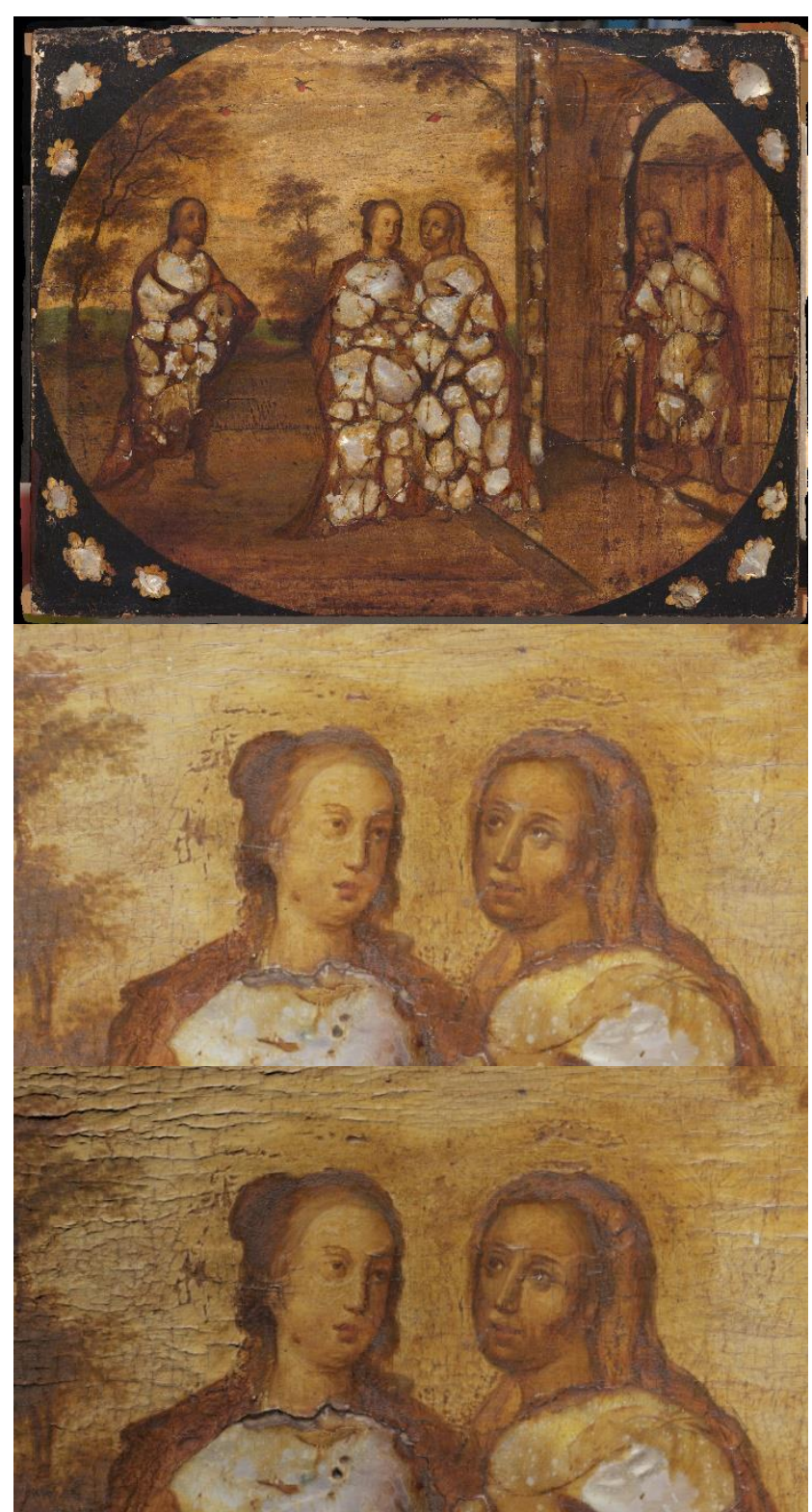

Figure 7 . The final orthophoto of the unnamed $17^{\text {th }}$-century painting (top), a rendered image of the colour orthophoto with a nadiral light (middle), a rendered image of the colour and depth with an angled light (bottom)

\section{REFERENCES}

Abate, D., Menna, F., Remondino, F., Gattari, M.G., 2014. 3D Painting Documentation: Evaluating of Conservation Conditions with 3D Imaging and Ranging Techniques. Int. Archives of Photogrammetry, Remote Sensing \& Spatial Information Sciences, Vol. XL-5, 1-8.

Agisoft PhotoScan, 2018. https://www.agisoft.com/, v. 1.4.3.

Aure, X., O’Dowd, P., Padfield, J., 2017. Generating 3D Models of Paintings through the Combination of 2D, 3D, and RTI Data. Electronic Visualisation and the Arts (EVA 2017). doi.org/10.14236/ewic/EVA2017.4

Berns, R.S., 2014. Camera Coding Evaluation for Image Archiving of Cultural Heritage. Rochester Institute of Technology, Rochester, New York, USA. 
Capturing Reality Reality Capture, 2018. https://www.capturingreality.com/, v. 1.0.3.

Dyer, J., Verri, G., Cupitt, J., 2013. Multispectral Imaging in Reflectance and Photo-induced Luminescene Modes: A User Manual. The British Museum, London.

Nocerino, E., Rieke-Zapp, D.H., Tinkl, E., Rosenbauer, R., Farella, E. M., Morabito, D., Remondino, F., 2018. Mapping VIS and UVL Imagery on 3D Geometry for Non-Invasive, NonContact Analysis of a Vase. Int. Archives of Photogrammetry, Remote Sensing \& Spatial Information Sciences, Vol. XLII-2, 773-780.

Remondino, F., Rizzi, A., Barazzetti, L., Scaioni, M., Fassi, F., Brumana, R. and Pelagotti, A., 2011. Review of Geometric and Radiometric Analyses of Paintings. The Photogrammetric Record, 26(136), 439-461.

Verhoeven, G., 2016. Basics of Photography for Cultural Heritage Imaging. In: Stylianidis, E., Remondino, F. (Eds.), 3D Recording, Documentation and Management of Cultural Heritage. Whittles Publishing, 127-251.

Verri, G., 2008. The use and distribution of Egyptian blue: a study by visible-induced luminescence imaging. In: K Uprichard \& A Middleton (eds), The Nebamun Wall Paintings. Archetype, London, pp. 41-50 .

Verri, G., 2009. The spatially resolved characterisation of Egyptian blue, Han blue and Han purple by photo-induced luminescence digital imaging. In: Analytical and Bioanalytical Chemistry, Vol 394(4), pp. 1011-1021.

https://doi.org/10.1007/s00216-009-2693-0

Warda, J. (Editor), Frey, F., Dawn Heller, D., Kushel, D., Vitale, T., Weaver, G., 2011. The AIC Guide to Digital Photography and Conservation Documentation, Third Edition. American Institute for Conservation of Historic and Artistic Works, Washington, D.C..

Westland, S., Ripamonti, C., Cheung, V., 2012. Computational Colour Science Using MATLAB, 2nd Edition. Wiley, Sussex, 411.

Wikimedia Commons contributors, 2004. Colour banding example01.png. Wikimedia Commons, the free media repository,

https://commons.wikimedia.org/w/index.php?title=File:Colour_ banding_example01.png\&oldid=129798746 (Last accessed March, 2019).

Wikimedia Commons contributors, 2014. CIE1931xy gamut comparison.svg. Wikimedia Commons, the free media repository,

https://commons.wikimedia.org/w/index.php?title=File:CIE193 1xy_gamut_comparison.svg\&oldid=324046804 (Last accessed March, 2019).

Wyszecki, G., Stiles, W.S., 1982. Color Science: Concepts and Methods, Quantitative Data and Formulae. Wiley, New York, 228-320.
APPENDIX

\begin{tabular}{|c|c|c|c|c|c|c|}
\hline & \multicolumn{3}{|c|}{ Photoscan (8-bit) } & \multicolumn{3}{c|}{ Reality Capture (8-bit) } \\
\hline & $\mathrm{R}$ & $\mathrm{G}$ & $\mathrm{B}$ & $\mathrm{R}$ & $\mathrm{G}$ & $\mathrm{B}$ \\
\hline$\mu_{\text {Error }}$ & 0 & 0 & 0 & -0.17 & -0.08 & -0.08 \\
\hline$\sigma_{\text {Error }}$ & 0 & 0 & 0 & 0.38 & 0.28 & 0.28 \\
\hline
\end{tabular}

Table 4. 8-bit colour errors (measured in tones) of a colour chart measured before and after processing the images in Agisoft Photoscan (2018) and Capturing Reality Capture (2018)

\begin{tabular}{|c|c|c|c|c|c|c|}
\hline & \multicolumn{3}{|c|}{ Photoscan (16-bit) } & \multicolumn{3}{c|}{ Reality Capture (8-bit) } \\
\hline & $\mathrm{R}$ & $\mathrm{G}$ & $\mathrm{B}$ & $\mathrm{R}$ & $\mathrm{G}$ & $\mathrm{B}$ \\
\hline$\mu$ Error & -0.75 & -0.71 & -3.13 & 26.83 & 21.83 & 27.50 \\
\hline$\sigma_{\text {Error }}$ & 5.32 & 3.21 & 8.00 & 27.49 & 11.96 & 36.38 \\
\hline
\end{tabular}

Table 5. 16-bit colour errors (measured in tones) of a colour chart measured before and after processing the images in Agisoft Photoscan (2018) and Capturing Reality Capture (2018)

\begin{tabular}{|c|c|c|c|c|c|c|}
\hline & \multicolumn{2}{|c|}{ Photoscan (16-bit) } & \multicolumn{3}{c|}{ Reality Capture (8-bit) } \\
\hline & $\mathrm{R}$ & $\mathrm{G}$ & $\mathrm{B}$ & $\mathrm{R}$ & $\mathrm{G}$ & $\mathrm{B}$ \\
\hline$\mu_{\text {Error }}$ & 0.42 & 1.33 & 0.38 & -0.17 & -0.13 & -0.04 \\
\hline$\sigma_{\text {Error }}$ & 4.13 & 1.83 & 4.68 & 0.38 & 0.34 & 0.20 \\
\hline
\end{tabular}

Table 6. 32-bit colour errors (measured in tones) of a colour chart measured as 16-bit images before and after processing the images in Agisoft Photoscan (2018) and Capturing Reality Capture (2018) as 32-bit images 